\title{
Attention-TCN-BiGRU: An Air Target Combat Intention Recognition Model
}

\author{
Fei Teng $(\mathbb{D}$, Yafei Song $* \mathbb{(})$ and Xinpeng Guo \\ Air and Missile Defense College, Air Force Engineering University, Xi'an 710051, China; \\ fei_teng0225@163.com (F.T.); afeu_gxp@163.com (X.G.) \\ * Correspondence: yafei_song@163.com
}

Citation: Teng, F.; Song, Y.; Guo, X. Attention-TCN-BiGRU: An Air Target Combat Intention Recognition Model. Mathematics 2021, 9, 2412. https://doi.org/10.3390/ math9192412

Academic Editors:

Ana Belén Nieto-Librero and Nerea González-García

Received: 6 September 2021

Accepted: 24 September 2021

Published: 28 September 2021

Publisher's Note: MDPI stays neutral with regard to jurisdictional claims in published maps and institutional affiliations.

Copyright: (c) 2021 by the authors. Licensee MDPI, Basel, Switzerland. This article is an open access article distributed under the terms and conditions of the Creative Commons Attribution (CC BY) license (https:// creativecommons.org/licenses/by/ $4.0 /)$.

\begin{abstract}
The prerequisite for victory in war is the rapid and accurate identification of the tactical intention of the target on the battlefield. The efficiency of manual recognition of the combat intention of air targets is becoming less and less effective with the advent of information warfare. Moreover, if the traditional method of combat intention of air targets is based only on data from a single moment in time, the characteristic information on the time-series data is difficult to capture effectively. In this context, we design a new deep learning method attention mechanism with temporal convolutional network and bidirectional gated recurrent unit (Attention-TCN-BiGRU) to improve the recognition of the combat intent of air targets. Specifically, suitable characteristics are selected based on the combat mission and air posture to construct a characteristic set of air target intentions and encode them into temporal characteristics. Each characteristic in the characteristic set is given an appropriate weight through the attention mechanism. In addition, temporal convolutional network (TCN) is used to mine the data for latent characteristics and bidirectional gated recurrent unit (BiGRU) is used to capture long-term dependencies in the data. Experiments comparing with other methods and ablation demonstrate that Attention-TCN-BiGRU outperforms state-of-the-art methods in terms of accuracy in recognizing target intent in the air.
\end{abstract}

Keywords: combat intention; bidirectional gated recurrent unit; attention mechanism; aerial target; temporal convolutional network

\section{Introduction}

With the development of military technology and aviation technology, informationization has gradually become the core of the modern battlefield, and future wars will also be informationized. At the same time, because the continuous development of modern technology has led to a dramatic increase in the dynamics and complexity of the environment of the modern battlefield, it has become increasingly difficult for military experts to identify the enemy's combat intentions in a timely and effective manner from the vast amount of information, especially from the air targets of large-scale electronic countermeasures [1]. Therefore, it is urgent to construct a decision-making aided combat system to improve the accuracy and speed of intention recognition of air target combat, so as to help decision-makers seize air superiority and even win the war.

In order to meet the needs of decision support system, many researchers have conducted research on the problem of intention identification, and air target tactical intention recognition has gradually become one of the research hotspots in modern air combat. The existing studies on the identification of tactical intentions against enemy targets mainly include evidence theory [2-4], template matching [5], expert systems [6], Bayesian networks [7-9], and neural networks [10-17]. The establishment of a standard template base of template matching technology, the collection of information and the construction of probability distribution function in evidence theory, the determination of the structure of Bayesian network and probability distribution parameters, and the construction of 
knowledge base and inference engine of the expert system all need to organize, abstract, and explicitly describe the empirical knowledge and knowledge representation of experts in related fields and the great difficulty of engineering implementation. References [10-14] use different deep learning methods, build and simulate the deep neural network of the human brain, and use data-driven methods to extract the semantic characteristics from the original data from low-level to high-level, from concrete to abstract, from general to specific, simulating the memory mechanism and reasoning mode of commander when judging the battlefield situation, overcoming the difficulties of traditional models in knowledge expression [15]. However, the target's tactical intention is realized through a series of tactical actions. Therefore, the dynamic attributes and battlefield environment of the target will show the characteristics of changing with time, and the enemy target has certain concealment and deception when performing combat operations. As a result, it is not sound enough for the above-mentioned deep learning method to judge the combat intention of the enemy target by using the characteristic information at a single time. In response to the drawbacks of the above methods, Liu et al. [16] established an air combat target intention prediction model based on long short-term memory (LSTM) network with incomplete information, introduced the cubic spline interpolation function fitting and the mean padding to repair the incomplete data, and used the adaptive moment estimation (Adam) optimization algorithm to accelerate the training speed of the target intention prediction model, so as to effectively prevent the local optimum problem. Xue et al. [17] designed panoramic convolutional long short-term memory neural network (PCLSTM), a new deep learning method, to improve the ability of intention recognition, and designed a time series pooling layer to reduce the parameters of the neural network. The above two methods based on LSTM network are verified by experiments to have a certain effect on air target tactical intention recognition. However, because LSTM is a one-way transmission network, it can only use past information to make judgments and cannot effectively use future information. At the same time, because the network shares the weight, the weight of different characteristics in identifying the combat intention of air targets is the same, which will have certain negative effects. Especially, when the intentions presented by different characteristics are contradictory, the weight proportion of key characteristics should be strengthened.

In view of the above problems, we propose an air target tactical intention recognition model based on Attention-TCN-BiGRU. Attention mechanism can learn the weight proportion of different characteristics to further highlight the key information affecting the intention, which has been successfully used in image caption generation, document classification, etc. In 2018, Bai et al. [18] proposed the model Temporal convolutional network (TCN), which has been shown to achieve state-of-the-art standards in many timing problems such as natural speech processing and audio synthesis. Compared with standard recurrent networks such as LSTM network and Gated Recurrent Unit (GRU), TCN has more accurate output results and a simpler structure. However, LSTM and GRU also have their advantages. TCN can effectively extract high-frequency and low-frequency information from the sequence, while LSTM and GRU are good at capturing long-term dependence in a series [19]. Therefore, our strategy is to explore the advantages of both networks rather than choose which one of them to use. In addition to this, we replace the GRU network with the Bidirectional Gated Recurrent Unit (BiGRU). BiGRU compares with GRU and TCN in its ability to learn not only from historical moment information but also from future moment information together for the current moment data [20]. The experimental results show that the Attention-TCN-BiGRU model is superior to other methods in the accuracy of intention recognition and has theoretical significance and reference value for auxiliary combat systems.

The rest of this paper is organized as follows: Section 2 describes in detail the air target intention recognition and how to select the characteristics and types of the tactical intention of air targets; Section 3 describes in detail the model proposed in this paper; Section 4 gives the experimental results and analysis; Section 5 concludes the paper. 


\section{Description of Aerial Target Combat Intention Recognition Problem}

Air target combat intention recognition is a process of judging the enemy's combat intentions. The state of enemy air targets will vary with different combat intentions. We use radar and other sensors to obtain the static attributes and real-time dynamic information of these targets from the complex battlefield environment. Combining the prior knowledge and the experience of experts, we analyze the data and propose predictions of combat intentions [11]. The target representation and reasoning process are shown in Figure 1.

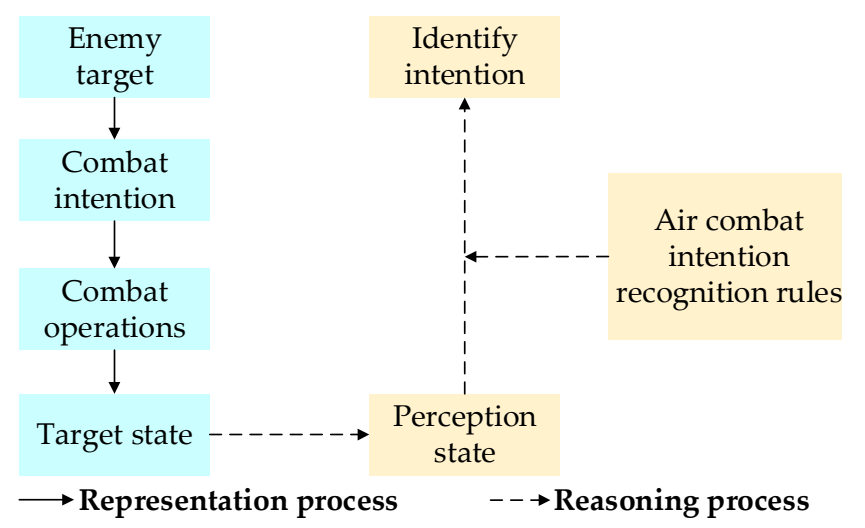

Figure 1. Hierarchical representation and reasoning process of intention.

Air target tactical intention recognition is a typical pattern recognition problem that can be described as a mapping of characteristics of air target tactical intention to types of air target tactical intention. Because the environment of the actual air battlefield is complex and changeable, and the air target will induce the decision-maker to wrong reasoning, it is easy to be deceived in identifying the enemy's tactical intention by relying on the characteristic information of the air target intention at a single moment. Therefore, it is more accurate and sounder to infer its tactical intention from the characteristic information of the enemy air target at multiple continuous moments [15]. Define $\boldsymbol{I}_{T}$ as the air combat target time series feature set composed of $T$ consecutive air combat real-time characteristic information from $t_{1}$ to $t_{T}$, that is, $\boldsymbol{I}_{T}=\left(\boldsymbol{I}^{\left(t_{1}\right)}, \boldsymbol{I}^{\left(t_{2}\right)}, \cdots, \boldsymbol{I}^{\left(t_{T}\right)}\right)$. Vector $\boldsymbol{Q}$ is defined as the space set of air target combat intention type, thereby determining the mapping function from the spatial set to the times series characteristic set of enemy air target tactical intention types as:

$$
\boldsymbol{Q}=f\left(\boldsymbol{I}_{T}\right)=f\left(\boldsymbol{I}^{\left(t_{1}\right)}, \boldsymbol{I}^{\left(t_{2}\right)}, \cdots, \boldsymbol{I}^{\left(t_{T}\right)}\right)
$$

To accurately identify the tactical intention of air targets, it is necessary to combine the tactical experience and military knowledge of experts in related fields and realize it through complex thinking activities such as key characteristic extraction, comparative analysis, association, and reasoning of air battlefield environment, so it is difficult to summarize it with simple mathematical formulas [13]. We extract the key characteristics of air targets to form the tactical intention characteristic set and use experts in related fields to label each sample to train the Attention-TCN-BiGRU network, thereby implicitly establishing the mapping relationship between the combat intention type space set and the time series characteristic data set. The whole process of air target combat intention recognition is shown in Figure 2.

As shown in Figure 2, the reasoning process [21,22] of air target tactical intention is as follows: Step 1-select and extract data information related to target tactical intention from real-time battlefield information, including battlefield environment information, target attribute information, and real-time state information of the target in the corresponding local time and space domain, and form an initial characteristic set; Step 2-Collect the state characteristic data of the target at $N$ consecutive moments $\left(\boldsymbol{I}^{\left(t_{n}\right)}-\boldsymbol{I}^{\left(t_{n+N}\right)}\right)$ and store them in a stack mode to form a characteristic vector set; Step 3-Integrate the characteristic data 
of $\left(\boldsymbol{I}^{\left(t_{n}\right)}-\boldsymbol{I}^{\left(t_{n+N}\right)}\right)$ moments, encode and normalize them to form a standardized temporal characteristic set; Step 4-Divide the times series characteristic set into the training set and testing set; Step 5-Input the training set to the Attention-TCN-BiGRU network training model; Step 6-Input the testing set to the trained air target intention recognition model output to get the intention recognition results.

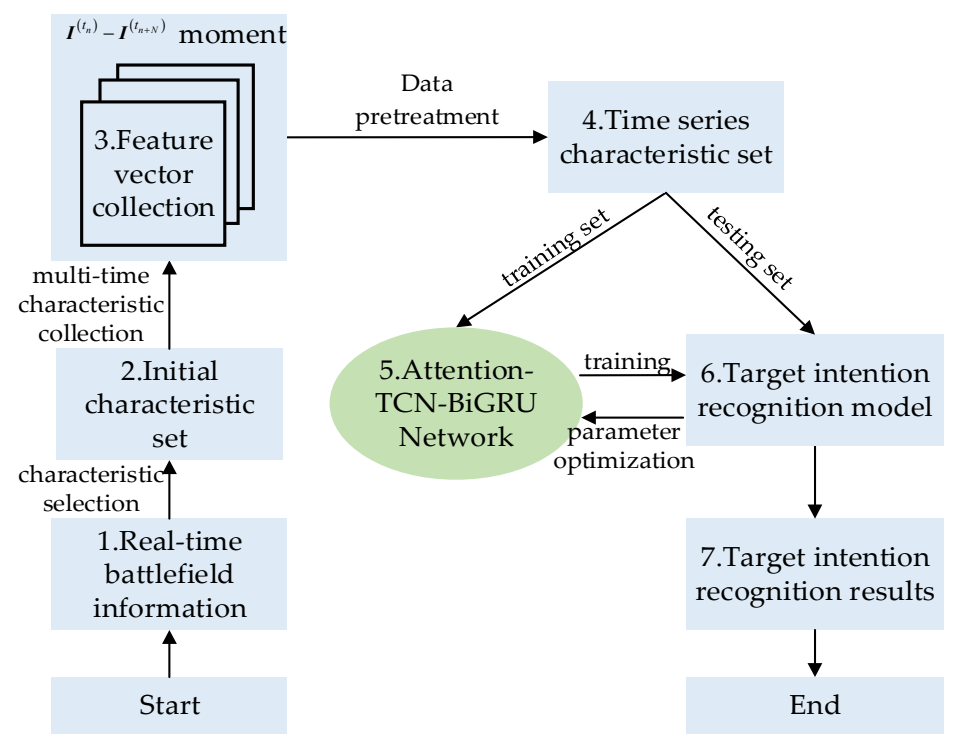

Figure 2. Process of air target tactical intention recognition.

\subsection{Selection of Tactical Intention Type}

For different combat forms, different enemy entities, and different setting contexts, there are differences in the target combat intention space set corresponding to the target combat intention set. Therefore, we need to define the combat intention space set of the enemy target according to the corresponding combat background, the attributes of the enemy target, and the possible combat missions. For example, in the target intention space set by Lu et al. [23], the potential threat of underwater target is \{evasion, patrol, and attack\}; Chen et al. [24] established the combat intent space set for the enemy's single-group maritime fleet as \{retreat, cover, attack, reconnaissance\}. In this paper, taking the enemy air target near-shore air attack on military buildings as the research background, we establish the tactical intention space set of enemy targets, including seven intention types \{attack, penetration, surveillance, reconnaissance, feint, retreat, and electronic interference\}. Please see Appendix A Table A1 for a detailed description of the seven intention types.

After determining the enemy's combat intention space set, how to transform the human cognitive pattern into labels that intelligent models can train and that correspond to the intention types in the combat intention space set is the key to applying the intelligent recognition model proposed in this paper to combat intention recognition [16]. Therefore, we can encapsulate the cognitive experience of experts in air combat into labels to train the intelligent recognition model of this paper. For the seven intention types in the enemy target tactical intention space established in this paper, $\{0,1,2,3,4,5,6\}$, a total of seven label values is set, respectively. The corresponding tactical intention type coding and model analysis mechanism are shown in Figure 3. For example, if the prediction result of intention output by the proposed model is 0 , it can be considered that the enemy target's combat intention against intended target is attacked. Therefore, using the above knowledge encapsulation and model analysis can clearly and easily describe human experience and knowledge, which is convenient for model training. 


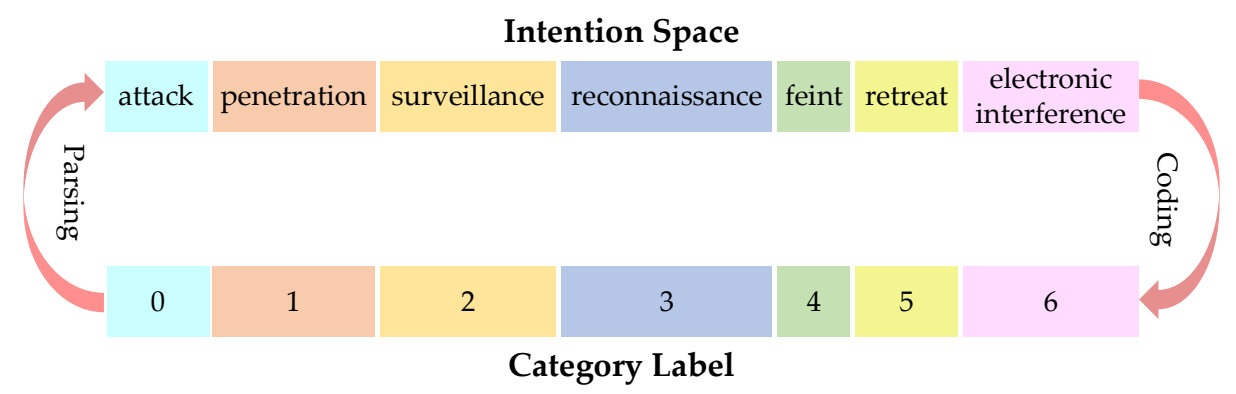

Figure 3. Schematic diagram of combat intention coding and analysis. The same color is a set of correspondences.

\subsection{Character Selection and Pre-Processing Process}

The air target's tactical intention is related to its combat mission and air situation. In order to identify different combat missions and air situations and to collect the actual characteristics of the sensors according to air targets such as radar stations, it is necessary to select appropriate air target intention characteristics.

From the perspective of a combat mission, when an air target performs a mission, some characteristic information needs to meet certain requirements. For example, when performing the task of penetration, it is divided into high-altitude penetration and lowaltitude penetration, with a corresponding height of $10 \mathrm{~km}-11 \mathrm{~km}$ and above $50 \mathrm{~m}-200 \mathrm{~m}$. Reconnaissance aircraft generally use low-altitude and high-altitude reconnaissance, with a corresponding height of $100 \mathrm{~m}-1000 \mathrm{~m}$ and above $15 \mathrm{~km}$. There is also a certain relationship between radar signal status of air targets and combat missions. For example, air-to-air radars are usually kept on during air combat, and air-to-air radars and marine radars are kept on during reconnaissance missions, and the radar status is coded so that 1 means the radar is on and 0 means the radar is off [11]. Different types of air targets have different application values and strategic significance. For example, fighter aircraft have strong offensiveness, and reconnaissance aircraft have strong mobile reconnaissance capabilities. Therefore, the type of enemy aircraft can also be used as the characteristic of combat intention. However, because it is difficult to directly identify the type of enemy aircraft, the radar cross-section (RCS) is used as an alternative to the type of enemy aircraft. RCS can represent the type of enemy aircraft to a certain extent. Under normal circumstances, the RCS of stealth aircraft and small aircraft is less than $1 \mathrm{~m}^{2}$, the RCS between $1 \mathrm{~m}^{2}$ and $10 \mathrm{~m}^{2}$ is a medium-sized aircraft, and the RCS greater than $10 \mathrm{~m}^{2}$ is a large-scale aircraft [17].

From the analysis of the air situation, many factors affect the superiority or inferiority of the air target. Considering the actual characteristics that can be collected, we mainly consider the enemy air target flight speed, altitude, acceleration, heading angle, azimuth angle, and the distance between the enemy, as shown in Figure 4. The air combat capability factor is also an important factor affecting the air situation. For the air combat capability of a fighter, a single machine air combat capability threat function is constructed according to the Reference [25]:

$$
C=\left[\ln \varepsilon_{1}+\ln \left(\varepsilon_{2}+1\right)+\ln \left(\sum \varepsilon_{3}+1\right)\right] \varepsilon_{4} \varepsilon_{5} \varepsilon_{6} \varepsilon_{7}
$$

In the formula, $\varepsilon_{1}-\varepsilon_{7}$ respectively represent fighter maneuverability, airborne weapon performance, airborne detection capability, fighter operation performance, fighter survivability, fighter combat range, and electronic information countermeasure capabilities. Air combat capability is an inherent attribute of aircraft. The air combat capability factor of enemy aircraft in a certain period can also be calculated by the above formula and then stored in the database and updated regularly according to the mastered information [26].

In addition, public opinion analysis is a worthy external characteristic to be selected [27]. When analyzing the target tactical intention, we often only focus on the attribute factors of the battlefield itself, while ignoring the influence of public opinion tendency in the war, which leads to inaccurate results of tactical intention analysis. There 
are many sources of public opinion, such as the government, the military, the Ministry of National Defense, the Ministry of Foreign Affairs, embassies, media, and civil organizations. Combining public opinion from different sources and considering the public opinion of combat environment of both sides, according to Miller's 9-level quantification theory [28], public opinion information can be quantified into 5 levels, including $1,3,5,7$, and 9 . To sum up, we have selected 12 kinds of characteristic information related to combating intention, including 8 kinds of numerical characteristics and 4 kinds of non-numerical characteristics. Tables 1 and 2 describe each characteristic in detail, showing 8 numerical data forms and 4 non-numerical data encoding forms of a sample at a certain time.

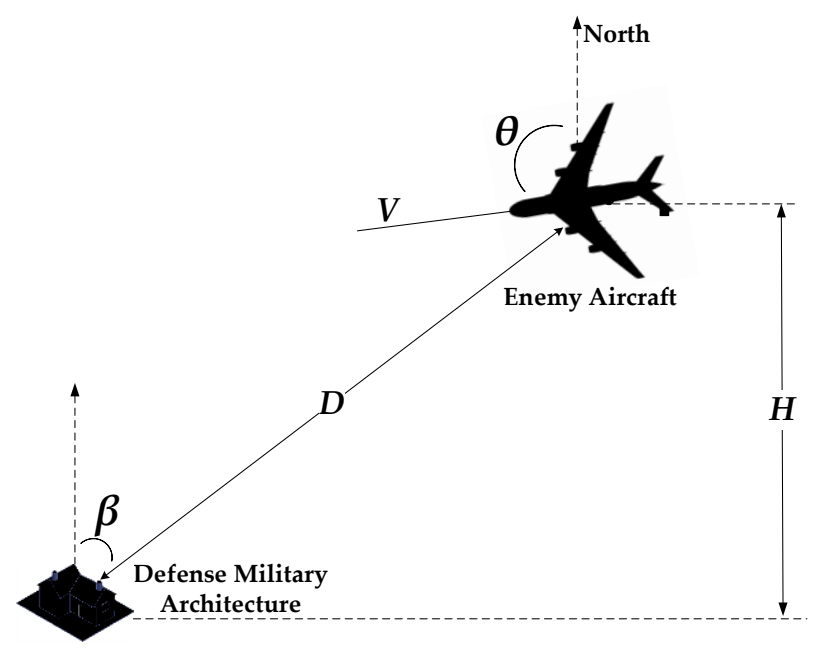

Figure 4. Relative geometric position of air combat. $H$ is the flight height, $D$ is the distance, $V$ is the flight speed, $\theta$ is the heading angle, and $\beta$ is the azimuth angle.

Table 1. Numerical air target intelligence data.

\begin{tabular}{|c|c|c|c|}
\hline Characteristic & Description & Unit & Numerical Value \\
\hline Distance & $\begin{array}{l}\text { the distance between enemy plane and } \\
\text { targeted building }\end{array}$ & $\mathrm{km}$ & 176.0 \\
\hline Velocity & the velocity of enemy plane & $\mathrm{m} / \mathrm{s}$ & 260.0 \\
\hline RCS & $\begin{array}{l}\text { the size of the radar reflection } \\
\text { cross-sectional area }\end{array}$ & $\mathrm{m}^{2}$ & 5.6 \\
\hline Height & the height of the enemy plane & $\mathrm{km}$ & 21.2 \\
\hline Azimuth angle & $\begin{array}{c}\text { the angle from targeted building to the } \\
\text { direction of the enemy aircraft (the true north is } \\
0 \text { mil, and the clockwise direction is divided } \\
\text { into } 6400 \text { mil) }\end{array}$ & mil & 1456.0 \\
\hline Acceleration & the acceleration of the enemy plane & $\mathrm{m} / \mathrm{s}^{2}$ & 5.6 \\
\hline Heading angle & $\begin{array}{l}\text { The flight direction of the enemy plane (the true } \\
\text { north is } 0 \text { degrees, and the clockwise direction } \\
\text { is } 360 \text { degrees) }\end{array}$ & $\circ$ & 45.3 \\
\hline Capability factor & the aerial combat capability of enemy aircraft & / & 9.4 \\
\hline
\end{tabular}

Because of the different units of various characteristics, their distribution ranges are quite different, and characteristics with a large variance will have a greater impact, which leads to the inability to accurately obtain core characteristics from the original data. In order to eliminate the influence of data dimension and improve the convergence efficiency and accuracy of the network, we now perform Min-Max Normalization on the above 12 air target combat intention characteristics. For the $i$-th type of numerical data $X_{i}=\left(x_{i 1}, x_{i 2}, \cdots x_{i r}, \cdots, x_{i k}\right)(i=1,2, \cdots, 12) ; k$ is the number of data of the $i$-th type. 
The result of mapping the $r$-th original data value $x_{i r}$ in the $i$-th characteristic to the interval $[0,1]$ is $x_{i r}^{\prime}$, and its formula is

$$
x_{i r}^{\prime}=\frac{x_{i r}-\min \boldsymbol{X}_{i}}{\max \boldsymbol{X}_{i}-\min \boldsymbol{X}_{i}}
$$

where $\min \boldsymbol{X}_{i}$ is the minimum value of the $i$-th characteristic $\boldsymbol{X}_{i} ; \max \boldsymbol{X}_{i}$ is the maximum value of the $i$-th characteristic $X_{i}$.

Table 2. Non-numerical air target intelligence data.

\begin{tabular}{ccc}
\hline Characteristic & Description & Categorical Value \\
\hline Electronic interference status & $\begin{array}{c}\text { whether the electronic jamming device is } \\
\text { turned on }\end{array}$ & 0 \\
\hline Air-to-air radar state & $\begin{array}{c}\text { whether the air-to-air radar is } \\
\text { switched on }\end{array}$ & 1 \\
\hline Air-to-surface radar state & $\begin{array}{c}\text { whether the air-to-surface radar is } \\
\text { switched on }\end{array}$ & 5 \\
\hline Public opinion analysis & $\begin{array}{c}\text { Current international public } \\
\text { opinion trends }\end{array}$ \\
\hline
\end{tabular}

\section{Model Framework}

In this section, we introduce our Attention-TCN-BiGRU model in detail. Attention mechanism assigns greater weight to key characteristics in the process of intention recognition. TCN extracts short-term local characteristics, whereas LSTM can capture the long-term dependence in a series. In addition, BiGRU can effectively use future information to make up for the shortcomings of the causal sequence in the TCN structure. To take full advantage of their merits, we combine them into a new hybrid model to further improve the accuracy of intention recognition. We introduce Attention mechanism, TCN, and BiGRU in Sections 3.1-3.3, respectively, and give the details of the overall framework of the proposed model in Section 3.4.

\subsection{Attention Mechanism}

Attention mechanism is a very hot topic at present, and there are various Attention mechanisms, such as Bottom-up Attention [29], Global attention [30], Self Attention [31], and so on. The essence of the attention mechanism comes from the human visual attention mechanism. When people perceive something visually, they generally do not see all of a scene from beginning to end but tend to observe and pay attention to a specific part of it according to their needs. Moreover, when people find that something they want to observe often appears in a certain part of a scene, they will learn and pay attention to that part when similar scenes appear in the future [32]. The distributed attention mechanism we use is a hierarchical attention network (HAN) proposed by Yang et al. [33] for text classification tasks. Not every word in a sentence is useful for classification. For example, when we do the emotion classification of text, we will focus on the words "happy" and "sad". In order to enable the recurrent neural network to automatically focus "attention" on these words, the author designs a word-based attention model. Firstly, the sentence composed of a sequence of words is transformed into a word vector through a word embedding matrix, and then the hidden layer output $\boldsymbol{h}_{i}$ is obtained through BiGRU network. Then a linear layer is transformed to obtain $\boldsymbol{u}_{i}$, using the softmax function to get the importance weight $\delta_{i}$ of each word, and finally, the information representation of the sentence vector $s$ is obtained by a weighted average of the output of BiGRU [34]. The formulas are as follows:

$$
\begin{gathered}
\boldsymbol{u}_{i}=\operatorname{Tanh}\left(\boldsymbol{W}_{w} \boldsymbol{h}_{i}+\boldsymbol{b}_{i}\right) \\
\delta_{i}=\frac{\exp \left(\boldsymbol{u}_{i}^{T} \boldsymbol{u}_{w}\right)}{\sum_{i} \exp \left(\boldsymbol{u}_{i}^{T} \boldsymbol{u}_{w}\right)}
\end{gathered}
$$




$$
\boldsymbol{s}=\sum_{i} \delta_{i} \boldsymbol{h}_{i}
$$

Formula (4) is a linear change process, $\boldsymbol{W}_{w}$ and $\boldsymbol{b}_{i}$ are the parameter vectors of the hidden layer; formula (5) is defined for softmax function, and $\boldsymbol{u}_{w}$ is a random initialization vector, which is continuously learned during training. formula (6) is the process of calculating sentence vector $s$.

In our proposed network, Attention mechanism is used to weight the contribution of different air target combat intention characteristics and extend the influence of the key characteristics, as shown in Figure 5. The implementation process is as follows. First, define the eigenvector $\boldsymbol{X}_{t}=\left(x_{1, t}, x_{2, t}, \cdots, x_{m, t}\right)$ at time step $t$, and calculate the proportion of characteristic weight according to the following softmax function formula.

$$
\alpha_{t}=\frac{\exp \left(\boldsymbol{X}_{t} \boldsymbol{u}_{w 1}\right)}{\sum \exp \left(\boldsymbol{X}_{t} \boldsymbol{u}_{w 1}\right)}
$$

where $\alpha_{t}=\left(\alpha_{1, t}, \alpha_{2, t}, \cdots, \alpha_{m, t}\right)$, and $\boldsymbol{u}_{w 1}$ is the hidden layer vector to be learned during the training process. Next, the weight ratio of the $i$-th characteristics is $\alpha_{i}=\left(\alpha_{i, t_{1}}, \alpha_{i, t_{2}}, \cdots, \alpha_{i, t_{n}}\right)$, and the average weight ratio is

$$
\alpha_{i}=\frac{1}{t_{n}} \sum_{t=t_{n}}^{t=t_{1}} \alpha_{i, t}
$$

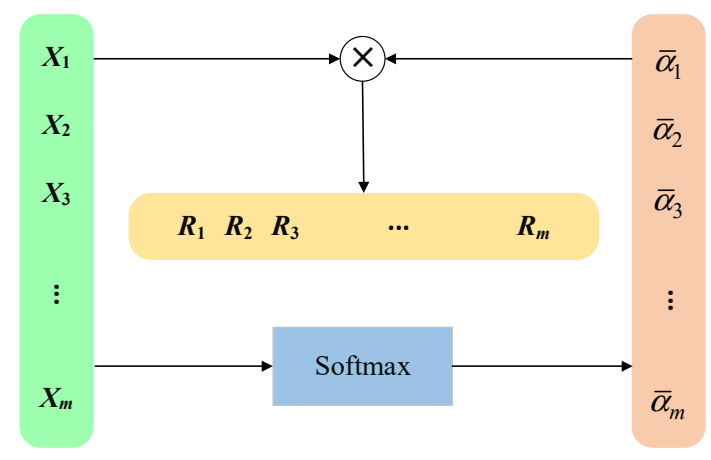

Figure 5. Attention mechanism structure used in this article. nism is

Finally, the weighted value of the $i$-th characteristics of the output of Attention mecha-

$$
\boldsymbol{R}_{i}=\alpha_{i} \boldsymbol{X}_{i}=\left(\alpha_{i} x_{i, t_{1}}, \alpha_{i} x_{i, t_{2}}, \cdots, \alpha_{i} x_{i, t_{n}}\right)
$$

\subsection{TCN}

Temporal convolutional network (TCN) is based on the convolutional neural network (CNN) model, and its structure combines causal convolution and dilated convolution. It can be used in a time series model, which can reduce the amount of calculation and keep a large receptive field for data. The proposed TCN effectively avoids the gradient disappearance or gradient explosion of RNN and has the advantages of parallel computing and controlling the sequence memory length by changing the size of the receptive field [35]. The following describes its structural components.

\subsubsection{Causal Convolutions}

Causal convolution can be visually represented in Figure 6 . The output at time $t$ is only related to the elements at time $t$ and before in the previous layer [36]. Different from the traditional convolutional neural network, causal convolution cannot see the future data, and it is a one-way structure, not a two-way one. That is to say, only with the previous cause can there be the subsequent result, which is a strict time constraint model, so it is called causal convolution. 


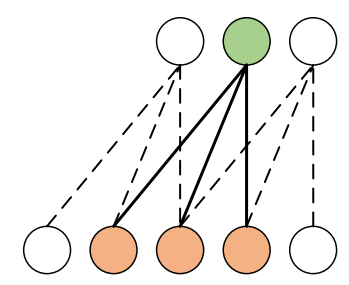

Figure 6. Causal convolution. Kernel size $=3$.

\subsubsection{Dilated Convolutions}

To address the problem of linear stacking of multiple convolutions in traditional neural networks to dilate the modeling of time series, TCN reduces the number of convolutional layers by using dilated convolutions to increase the range of the receptive field of each layer [37]. The difference between dilated convolutions and ordinary convolutions is that dilated convolution allows interval sampling of input during convolution, and the sampling rate depends on dilation factors. The formula of the receptive field is

$$
R F=(k-1) * d+1
$$

where $k$ is the kernel size, and $d$ is the dilation factor. There are two ways to increase the TCN receptive field: increasing the dilation factor and choosing a larger kernel size, as shown in Figure 7. In the operation of dilation convolution, the dilation factor will increase exponentially with the increase of network depth, so the convolution network can still obtain a large receptive field with fewer layers [38].

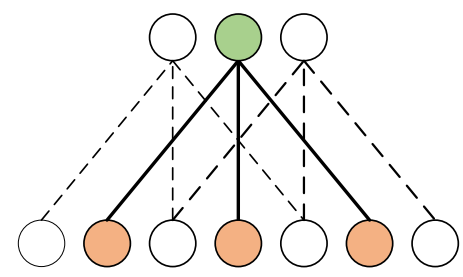

Figure 7. Dilated convolution. Dilation factors $=2$, kernel size $=3$.

\subsubsection{Residual Connections}

Another important network structure in the TCN network is the residual module. The residual module, shown in Figure 8, contains two layers of dilation causal convolution and nonlinear mapping. Moreover, the residual network has added the identity mapping of cross-layer connections, so that the network can transmit information in a cross-layer manner, which can solve the problem of gradient disappearance and make the shallow network easy to dilate into the deep network [39]. At the same time, Dropout and batch normalization are added to prevent the model from overfitting and speeding up training.

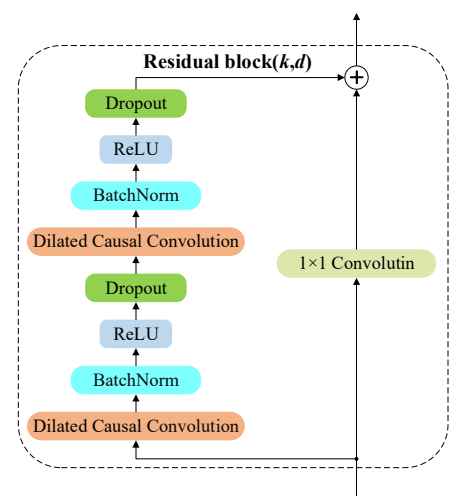

Figure 8. TCN residual block. $k$ is the kernel size, and $d$ is the dilation factor. 


\subsection{BiGRU}

As a variant of Recurrent Neural Network (RNN), Gated Recurrent Unit (GRU) has a recursive structure similar to RNN and has the "memory" function of processing time-series data. Meanwhile, GRU can effectively alleviate the gradient disappearance and gradient explosion that may occur during RNN training, thus effectively solving the problem of long-term memory. LSTM network is also a variant of RNN [40]. Its performance is almost the same as that of GRU, but GRU is simpler in structure, which can reduce the amount of calculation and improve the training efficiency [41]. The internal structure of GRU is shown in Figure 9. GRU has two inputs: the output state $\boldsymbol{h}_{t-1}$ of the previous time and the input sequence value $\boldsymbol{x}_{t}$ of the current time, and the output is the state $\boldsymbol{h}_{t}$ of the current time. It mainly updates the model state through resetting gate $\boldsymbol{r}_{t}$ and updating gate $z_{t}$, and the gate $r_{t}$ reset controls the degree of forgetting of historical state information, so that the network can lose unimportant information. The gate $z_{t}$ update controls the proportion of the previous state information brought into the current state, helping the network to remember long-term information [42]. Its internal calculation formulas are as follows:

$$
\begin{gathered}
\boldsymbol{r}_{t}=\sigma\left(\boldsymbol{W}_{r} \boldsymbol{x}_{t}+\boldsymbol{U}_{r} \boldsymbol{h}_{t-1}\right) \\
\boldsymbol{z}_{t}=\sigma\left(\boldsymbol{W}_{z} \boldsymbol{x}_{t}+\boldsymbol{U}_{z} \boldsymbol{h}_{t-1}\right) \\
\widetilde{\boldsymbol{h}}_{t}=\operatorname{Tanh}\left(\boldsymbol{W}_{\widetilde{h}} \boldsymbol{x}_{t}+\boldsymbol{U}_{\widetilde{h}}\left(\boldsymbol{r}_{t} \odot \boldsymbol{h}_{t-1}\right)\right) \\
\boldsymbol{h}_{t}=\left(1-\boldsymbol{z}_{t}\right) \odot \boldsymbol{h}_{t-1}+\boldsymbol{z}_{t} \odot \widetilde{\boldsymbol{h}}_{t}
\end{gathered}
$$

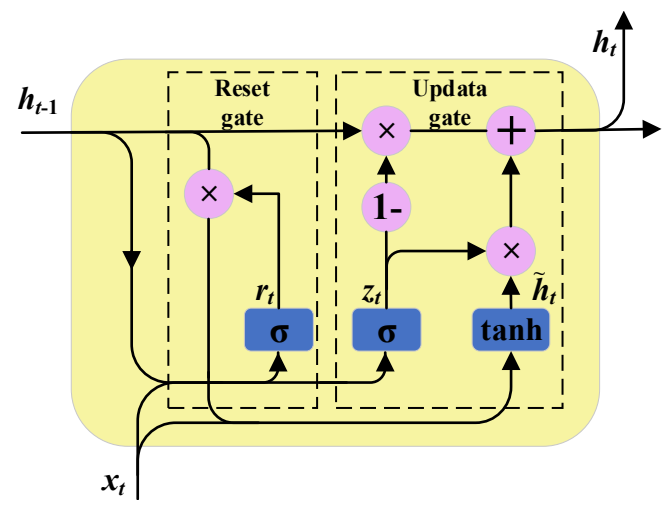

Figure 9. Gated recurrent unit structure.

In the formulas and in Figure 9, $\sigma$ is the sigmoid activation function, which changes the intermediate state to the range of $[0,1] ; \boldsymbol{h}_{t-1}$ and $\boldsymbol{h}_{t}$ are the output states at moments $t-1$ and $t$, respectively; $x_{t}$ is the input sequence value at moment $t ; \widetilde{h}_{t}$ is the candidate output state. $\boldsymbol{W}_{r}, \boldsymbol{W}_{z}, \boldsymbol{W}_{h_{h}} \boldsymbol{U}_{r}, \boldsymbol{U}_{z}$ and $\boldsymbol{U}_{\tilde{h}}$ are the weight coefficient matrix corresponding to each part; Tanh is the hyperbolic tangent function; $\odot$ is the Hadamard product of the matrix.

The traditional GRU structure usually spreads in one direction along with the sequence transmission, and the information it obtains is the historical information before the current time, which leads to ignoring the future information. However, BiGRU structure is composed of forward GRU and backward GRU, which can capture the characteristics of front and back information [43]. Its model structure is shown in Figure 10.

As can be seen from Figure 10, the BiGRU hidden layer state $\boldsymbol{h}_{t}$ at the moment $\mathrm{t}$ can be found in two parts: the forward hidden layer state $\vec{h}_{t}$ and the backward hidden layer state $\overleftarrow{\boldsymbol{h}}_{t}$. The forward hidden layer state $\overrightarrow{\boldsymbol{h}}_{t}$ is determined by the current input $\boldsymbol{x}_{t}$ and the forward hidden layer state $\overrightarrow{\boldsymbol{h}}_{t-1}$ at moment $t-1$. The backward hidden layer state $\overleftarrow{\boldsymbol{h}}_{t}$ is determined by the current input $x_{t}$ and the backward hidden layer state $\overrightarrow{\boldsymbol{h}}_{t-1}$ at 
moment $t+1$. The calculation formulas are as follows, where $w_{i}(i=1,2 \ldots, 6)$ represents the weight from one unit layer to another.

$$
\begin{gathered}
\overrightarrow{\boldsymbol{h}}_{t}=f\left(\boldsymbol{w}_{1} \boldsymbol{x}_{t}+\boldsymbol{w}_{2} \overrightarrow{\boldsymbol{h}}_{t-1}\right) \\
\overleftarrow{\boldsymbol{h}}_{t}=f\left(\boldsymbol{w}_{3} \boldsymbol{x}_{t}+\boldsymbol{w}_{5} \overleftarrow{\boldsymbol{h}}_{t+1}\right) \\
\boldsymbol{h}_{t}=g\left(\boldsymbol{w}_{4} \overrightarrow{\boldsymbol{h}}_{t}+\boldsymbol{w}_{6} \overleftarrow{\boldsymbol{h}}_{t}\right)
\end{gathered}
$$

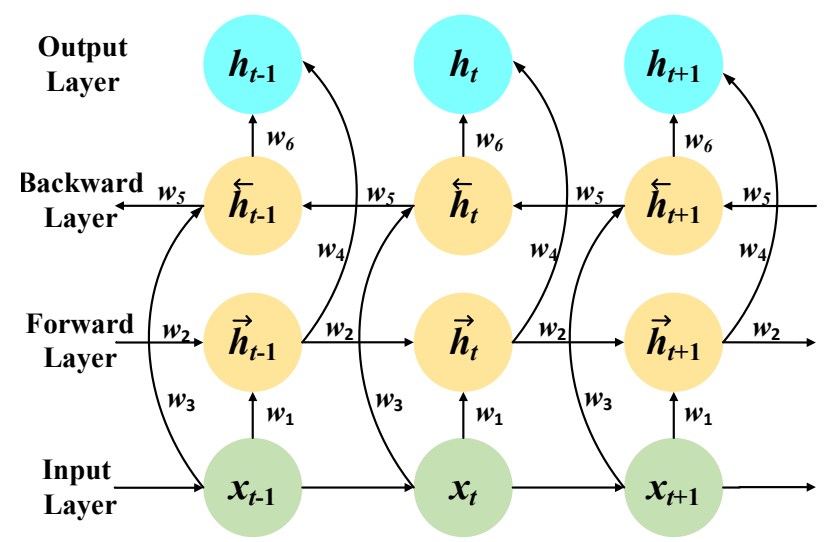

Figure 10. Bidirectional gated recurrent unit structure.

\subsection{Attention-TCN-BiGRU}

Our strategy is to make full use of these three algorithms to solve our problems: the ability of Attention mechanism to select key characteristics, the ability of TCN to extract characteristics, the ability of BiGRU to capture long-term dependence, and the ability to obtain future information. Therefore, the model is named Attention-TCN-BiGRU. Figure 11 illustrates the general framework of our proposed model.

The input of the model is the data $X$ after data preprocessing in Section 2.2, and its shape is (sample size, time step, characteristic size). In the first stage, the characteristic vector $\boldsymbol{X}_{i}$ of each category in $\boldsymbol{X}$ are given different weights $\alpha_{i}(i=1,2, \cdots, m)$ through the attention mechanism in Section 3.1. In the second stage, we perform characteristic extraction over $\boldsymbol{T}_{j}=\left(\alpha_{1} x_{1, t_{j}}, \alpha_{2} x_{2, t_{j}}, \cdots, \alpha_{m} x_{m, t_{j}}\right)(j=1,2, \cdots, n)$ through TCN, which consists of two residual blocks. The first residual module consists of two dilation causal convolution layers, with kernel size of 4 , dilation factor of 1 , and filter number of 7 . The dilation factor of the second residual module is 2 , and the remaining parameters are the same as those of the first residual module. In the third stage, we use the output vector $T_{j}{ }^{\prime}$ of the TCN as the input of the BiGRU network to extract the long-term correlation in the time series and the correlation between future information and current information. We take the output $\boldsymbol{h}_{t_{64}}$ at the last moment of the second layer of BiGRU network as the input of the full connection layer and output the intention type label with the highest probability by the following formula.

$$
y=\operatorname{softmax}\left(\boldsymbol{w} \boldsymbol{h}_{t_{64}}+\boldsymbol{b}\right)
$$

where $y$ is the intention type label, $w$ is the weight matrix to be learned, and $\boldsymbol{b}$ is the bias vector. 


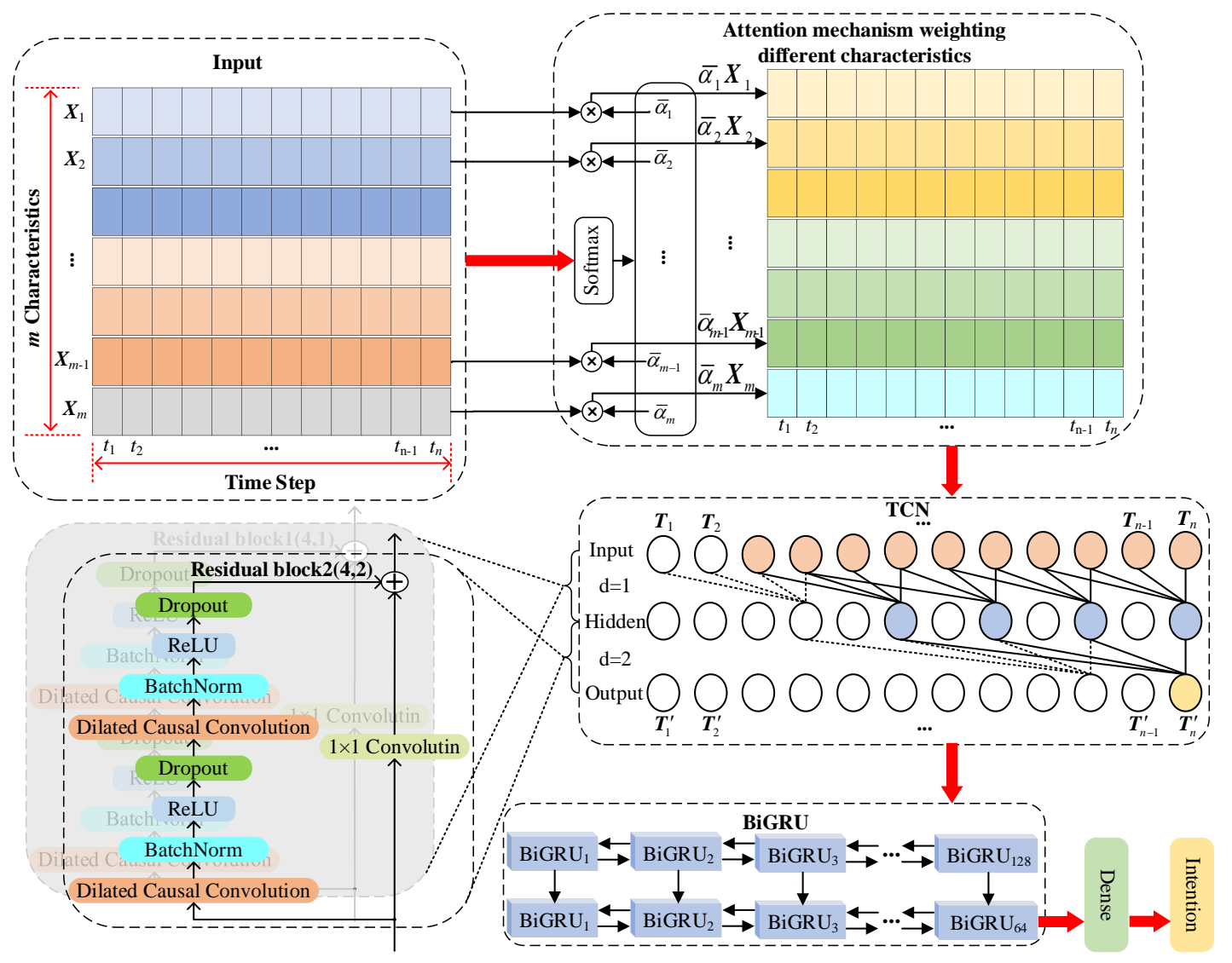

Figure 11. Proposed Attention-TCN-BiGRU framework.

\section{Experimental Analysis}

\subsection{Experimental Data and Environment}

The experimental data were obtained from the Air Combat Maneuvering Instrumentation (ACMI), whose labels were revised by experts in the field of air warfare. There were 10,500 samples in total, including 8400 training samples and 2100 test samples, with $14.3 \%$ of intentions per category. Time step was 12, and characteristic size was 12 .

The experiments were conducted using the Python 3.8 language, in a Quadro RTX 5000/PCle/SSE2 GPU and CUDA11.0 accelerated environment, using the Keras 2.4.3 deep learning framework, with a computer configuration of an x86-64 CentOS7 PC system, Intel ${ }^{\circledR}$ Xeon(R) Sliver 4110 CPU @ 2.10GHz, and 64GB of RAM. The number of epochs set for the experiment was 100, and the batch size was 256 .

\subsection{Evaluation Metric}

We propose the performance of the Attention-TCN-BiGRU air target recognition model of combat intention for validation. Five metrics are used to evaluate the classification of the network, namely, Loss, Accuracy, Precision, Recall, and F1-score. They are calculated as follows.

$$
\begin{gathered}
\text { Loss }=-\sum_{i=1}^{i=q} y_{i 1} \log \hat{y}_{i 1}+y_{i 2} \log \hat{y}_{i 2}+\cdots+y_{i d} \log \hat{y}_{i d} \\
\text { Accuracy }=\frac{T P+T N}{T P+F N+F P+T N} \\
\text { Precision }=\frac{T P}{T P+F P} \\
\text { Recall }=\frac{T P}{T P+F N}
\end{gathered}
$$




$$
\text { F1 score }=\frac{2 \times \text { Precision } \times \text { Recall }}{\text { Precision }+ \text { Recall }}
$$

where $q$ is the number of samples, $d$ is the number of categories, $y_{i d}$ is the one-hot coded value $(0$ or 1$), \hat{y}_{i d}$ is the output value of the Softmax function $\left(\sum_{d=1}^{d=7} \hat{y}_{i d}=1\right), T P$ is the True Positive, FN is the False Negative, FP is the False Positive, and TN is the True Negative.

\subsection{Parameter Tuning}

There are also many hyperparameters in Attention-TCN-BiGRU that have a significant impact on intention recognition performance. These include Filter numbers and Kernel size in the TCN network, Neurons size in the BiGRU network, Optimizer, and Learning rate of the whole network.

Appropriate size and number of convolutional kernels help to learn the rich characteristics in the data. As can be seen in Tables 3 and 4, the highest accuracy and lowest loss values are achieved when Filter numbers $=7$ and Kernel size $=4$, so they are set to 7 and 4 , respectively.

Table 3. Comparison of different filter numbers in the Attention-TCN-BiGRU.

\begin{tabular}{ccc}
\hline Filter Numbers & Accuracy & Loss \\
\hline 5 & 93.2 & 0.186 \\
7 & 94.6 & 0.138 \\
10 & 93.6 & 0.171 \\
15 & 92.5 & 0.207 \\
20 & 92.3 & 0.238 \\
\hline
\end{tabular}

Table 4. Comparison of different kernel sizes in the Attention-TCN-BiGRU.

\begin{tabular}{ccc}
\hline Kernel Size & Accuracy & Loss \\
\hline 2 & 92.5 & 0.209 \\
3 & 93.7 & 0.172 \\
4 & 94.6 & 0.138 \\
5 & 92.6 & 0.210 \\
6 & 92.3 & 0.227 \\
\hline
\end{tabular}

The number of neuron nodes is not as good as possible. Too many neuron nodes are prone to "overfitting". The appropriate number of BiGRU neurons has a significant impact on the intention recognition performance of Attention-TCN-BiGRU. We considered both single-layer and two-layer BiGRU networks and tried different numbers of neurons. As can be seen from Table 5, the highest accuracy and lowest loss values were achieved when the BiGRU was set to two layers with 128 and 64 neurons, respectively.

Table 5. Comparison of different neurons size in the Attention-TCN-BiGRU.

\begin{tabular}{ccc}
\hline Number of Neurons & Accuracy & Loss \\
\hline 128 & 94.0 & 0.162 \\
256 & 94.0 & 0.170 \\
512 & 92.3 & 0.201 \\
6464 & 93.7 & 0.185 \\
12864 & 94.6 & 0.138 \\
128128 & 93.3 & 0.181 \\
\hline
\end{tabular}

Regarding optimizers, we compared five candidate algorithms; Stochastic Gradient Descent (SGD) [44], Root Mean Square prop (RMSprop) [45], Adaptive Moment Estimation (Adam) [46], Adamax [46], and Nesterov-accelerated Adaptive Moment Estimation (Nadam) [47] were used. As can be seen from Table 6, the Nadam optimizer has the highest 
accuracy and lowest loss values compared to the other optimizers. Therefore, Nadam was chosen for the optimization of our model. The default learning rate of Nadam in the Keras 2.4.3 deep learning framework is 0.002, which we have adjusted. As can be seen from Table 7 , the accuracy and loss value of intention recognition is optimal when the learning rate is 0.004 , so the learning rate we choose is 0.004 .

Table 6. Comparison of the different optimizers in the Attention-TCN-BiGRU.

\begin{tabular}{ccc}
\hline Optimizer & Accuracy & Loss \\
\hline Nadam & 94.6 & 0.138 \\
Adam & 94.3 & 0.151 \\
Adamax & 92.0 & 0.203 \\
RMSprop & 94.1 & 0.159 \\
SGD & 90.1 & 0.276 \\
\hline
\end{tabular}

Table 7. Comparison of different learning rates in the Attention-TCN-BiGRU.

\begin{tabular}{ccc}
\hline Learning Rate & Accuracy & Loss \\
\hline 0.002 & 93.9 & 0.153 \\
0.004 & 94.6 & 0.138 \\
0.006 & 93.2 & 0.172 \\
0.008 & 92.8 & 0.197 \\
0.010 & 91.0 & 0.231 \\
\hline
\end{tabular}

The parameter settings for each network layer of our Attention-TCN-BiGRU model are shown in Table 8.

Table 8. Parameters of each layer of the proposed method.

\begin{tabular}{|c|c|c|}
\hline & Layers & Parameters \\
\hline $\begin{array}{l}\text { Residual } \\
\text { block } 1\end{array}$ & $\begin{array}{l}\text { Conv1D layer } \\
\text { Activation function } \\
\text { Normalization method } \\
\text { Conv1D layer } \\
\text { Activation function } \\
\text { Normalization method } \\
\text { Conv1D layer }\end{array}$ & $\begin{array}{l}\text { Filter numbers }=7, \text { Kernel size }=4 \text {, Dilation_rate }=1 \text {, Padding = 'causal' } \\
\text { Rectified Linear Unit } \\
\text { Batch Normalization } \\
\begin{array}{r}\text { Filter numbers }=7, \text { Kernel size }=4 \text {, Dilation_rate }=1 \text {, Padding = 'causal' } \\
\text { Rectified Linear Unit } \\
\text { Batch Normalization }\end{array} \\
\text { Filter numbers }=7, \text { Kernel size }=1 \text {, Dilation_rate }=1 \text {, Padding = 'same' }\end{array}$ \\
\hline $\begin{array}{l}\text { Residual } \\
\text { block } 2\end{array}$ & $\begin{array}{l}\text { Conv1D layer } \\
\text { Activation function } \\
\text { Normalization method } \\
\text { Conv1D layer } \\
\text { Activation function } \\
\text { Normalization method } \\
\text { Conv1D layer }\end{array}$ & $\begin{array}{l}\begin{array}{l}\text { Filter numbers }=7, \text { Kernel size }=4 \text {, Dilation_rate }=2 \text {, Padding = 'causal' } \\
\text { Rectified Linear Unit } \\
\text { Batch Normalization }\end{array} \\
\begin{array}{r}\text { Filter numbers }=7, \text { Kernel size }=4 \text {, Dilation_rate }=2 \text {, Padding = 'causal' } \\
\text { Rectified Linear Unit } \\
\text { Batch Normalization }\end{array} \\
\text { Filter numbers }=7, \text { Kernel size }=1 \text {, Dilation_rate }=2, \text { Padding = 'same' }\end{array}$ \\
\hline & $\begin{array}{l}\text { Bidirectional GRU } 1 \\
\text { Bidirectional GRU } 2 \\
\text { Dense }\end{array}$ & $\begin{array}{c}\text { Hidden size }=128 \\
\text { Hidden size }=64 \\
\text { Hidden size }=7 \text {, activation }=\text { 'softmax' }\end{array}$ \\
\hline
\end{tabular}

\subsection{Results and Analysis}

4.4.1. Attention-TCN-BiGRU Intention Recognition Result Analysis

The experimental results of the Attention-TCN-BiGRU model are shown in Figure 12. The convergence reached around 40 epochs, and the accuracy of the test set fluctuated around $94 \%$ and could reach up to $94.6 \%$, with a loss value around 0.15 which could reach as low as 0.138 . 


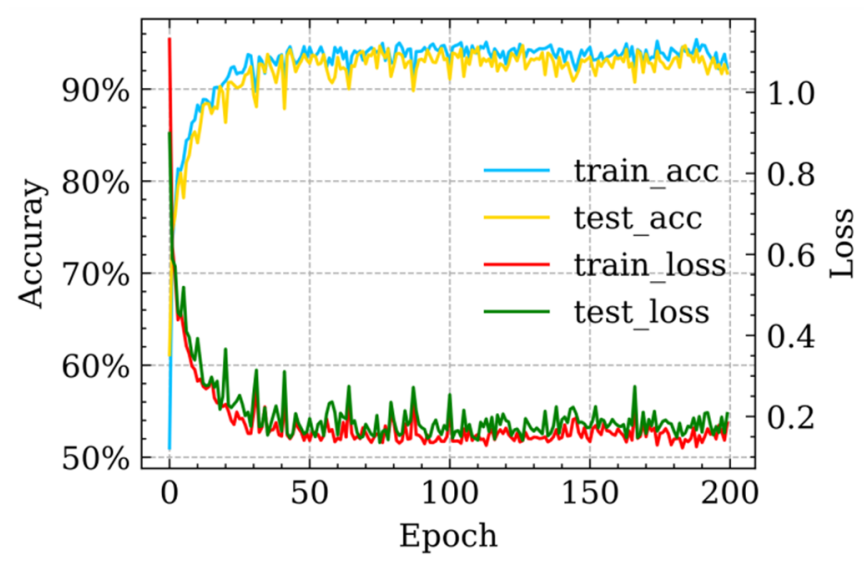

Figure 12. Accuracy and loss value of the Attention-TCN-BiGRU model test set and training set.

The confusion matrix for the test set was produced to allow further observation of the relationship between the recognition intentions. The diagonal line indicates the number of correctly identified samples, as shown in Figure 13. As can be seen from Figure 13, the accuracy of the model is high for all seven intentions, with the highest accuracy of $98.7 \%$ for retreat and the lowest accuracy of $88.7 \%$ for the feint. In addition, it can be found that there are some mutual recognition errors for attack and feint intentions and a few mutual recognition errors for reconnaissance and surveillance. The network was unable to learn the obvious difference between the two intentions, which led to the misrecognition of the two intentions, in line with the actual situation.

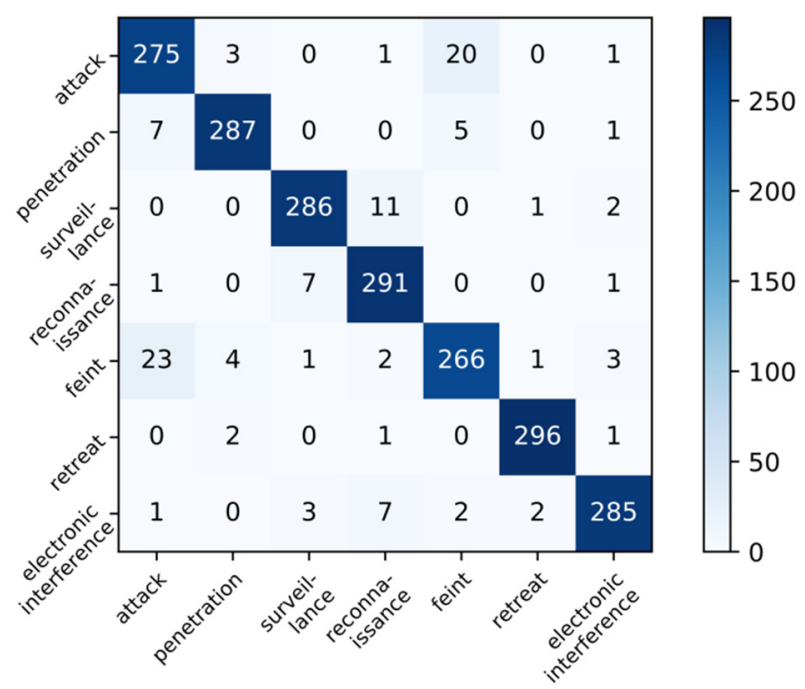

Figure 13. Confusion matrix of Attention-TCN-BiGRU intention recognition results.

\subsubsection{Analysis of Characteristic Weight Proportion}

The weights assigned to the aerial target characteristic set after the Attention layer are now analyzed in order to verify the effectiveness of the Attention mechanism. Its results are shown in Figure 14, with 12 characteristics on the horizontal axis and 7 intentions on the vertical axis.

From Figure 14, it is derived that the four characteristics of distance, altitude, speed and heading angle received the most attention among the characteristics. By reviewing the relevant reference $[48,49]$ and consulting with relevant experts, it is clear that angle and altitude play the most critical role in the execution of the mission by warplanes, which is consistent with the experimental results. Combined with the analysis of the confusion matrix in Figure 14, there exist three types of intentions with more mutual recognition errors, attack, feint, and penetration, and many degrees of similarity in the attention given 
to various types of characteristics in their Attention layer, and the same situation exists for surveillance and reconnaissance. The two corroborate each other, further demonstrating that the Attention mechanism is indeed effective in focusing on key characteristics.

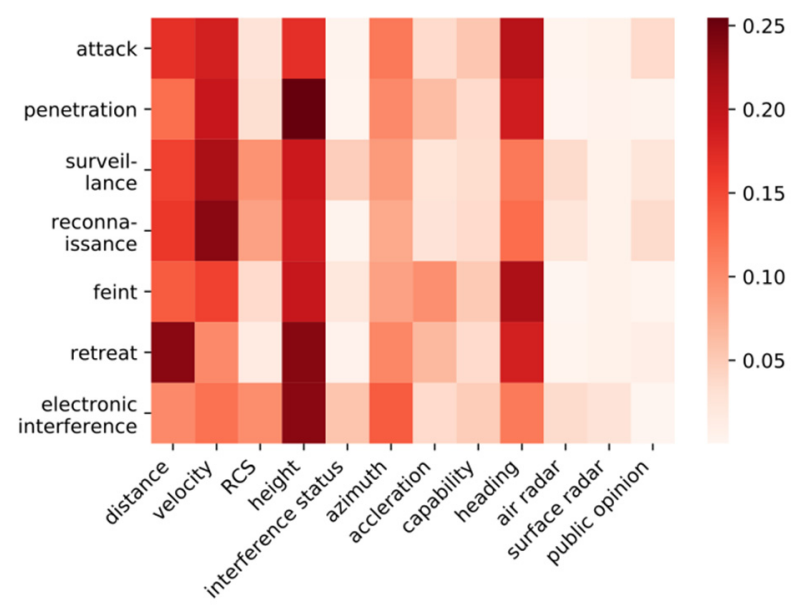

Figure 14. Attention mechanism assigns weight to characteristics.

\subsubsection{GRU, LSTM, BiLSTM, and BiGRU Selection Experiments}

The Attention-TCN network was combined with BiGRU, BiLSTM, GRU, and LSTM, respectively, in order to verify that using the BiGRU network was superior, and the experimental results are shown in Table 9. The intention recognition accuracy of AttentionTCN-BiGRU and Attention-TCN-BiLSTM were similar, and both were higher than that of Attention-TCN-GRU and Attention-TCN-LSTM networks, thus verifying that the introduction of bidirectional propagation mechanism can effectively improve the intention recognition accuracy. In terms of intention recognition time for a single sample, the lowest time was $43.2 \mathrm{~ms}$ for Attention-TCN-GRU, and the longest time was $54.2 \mathrm{~ms}$ for AttentionTCN-BiLSTM, because the LSTM structure consists of four parts and the GRU structure consists of three parts, and the number of GRU parameters is about one quarter less than that of LSTM. Therefore, we chose the Attention-TCN-BiGRU network because it has lower time complexity when the performance is similar to Attention-TCN-BiLSTM.

Table 9. Comparison of four models.

\begin{tabular}{cccc}
\hline Model & Accuracy (\%) & Loss & Time (ms) \\
\hline Attention-TCN-BiGRU & 94.6 & 0.138 & 50.4 \\
Attention-TCN-BiLSTM & 94.7 & 0.129 & 54.2 \\
Attention-TCN-GRU & 93.9 & 0.148 & 43.2 \\
Attention-TCN-LSTM & 94.1 & 0.151 & 46.9 \\
\hline
\end{tabular}

\subsubsection{Comparative Analysis of Intention Recognition Methods}

In order to verify the superiority of our proposed method, we now compare it with Zhou et al. [11] proposed the use of ReLU function and Adam algorithm to optimize the deep back propagation (DBP) neural network air target combat intention recognition model; Zhai et al. [12] proposed a standardized fully connected residual (BN-FC-RES) network model for the analysis of air target combat intentions; Liu et al. [13] proposed a target intention recognition model based on radial basis function (RBF) neural network; Ou et al. [14] proposed a stack autoencoder (SAE) tactical intent intelligent recognition model; Liu et al. [16] proposed a long-short-term memory (LSTM) network-based air combat target intention prediction model under incomplete information, and Xue et al. [17] proposed a panoramic convolutional long short-term memory network (PCLSTM) for air target combat intention recognition. The experimental results are shown in Table 10. 
Table 10. Comparison of different intention recognition models.

\begin{tabular}{ccc}
\hline Model & Accuracy $\mathbf{( \% )}$ & Loss \\
\hline Attention-TCN-BiGRU & 94.6 & 0.138 \\
PCLSTM & 88.7 & 0.338 \\
LSTM & 84.5 & 0.445 \\
BN-FC-RES & 81.6 & 0.498 \\
SAE & 76.8 & 0.553 \\
DBP & 75.9 & 0.548 \\
RBF & 71.3 & 0.632 \\
\hline
\end{tabular}

\subsubsection{Ablation Experiment}

A comparative experimental analysis was conducted through ablation experiments and three assessment metrics; Precision, Recall, and F1 Score, were introduced to assess each type of intention in order to further demonstrate the superiority of our proposed method. The experimental results are shown in Tables 11 and 12 and Figure 15.

Table 11. Results of ablation experiment.

\begin{tabular}{ccccc}
\hline \multicolumn{3}{c}{ Model Composition Structure } & \multirow{2}{*}{ Accuracy (\%) } & Loss \\
\cline { 1 - 2 } Attention & TCN & BiGRU & & 0.138 \\
\cline { 1 - 2 } & $\sqrt{ }$ & $\sqrt{ }$ & 94.6 & 0.185 \\
$\sqrt{ }$ & $\sqrt{ }$ & $\sqrt{ }$ & 93.3 & 0.173 \\
$\sqrt{ }$ & & $\sqrt{ }$ & 93.5 & 0.247 \\
& $\sqrt{ }$ & & 90.8 & 0.298 \\
& $\sqrt{ }$ & $\sqrt{ }$ & 90.2 & 0.435 \\
\hline
\end{tabular}

Table 12. Results of evaluation indexes of ablation experiment. (1), (2), (3), (4), (5), and (6) respectively represent the AttentionTCN-BiGRU, Attention-TCN, Attention-BiGRU, TCN-BiGRU, TCN, and BiGRU air target intention recognition models.

\begin{tabular}{cccccccccccccccccccc}
\hline \multirow{2}{*}{$\begin{array}{c}\text { Evaluation Index } \\
\text { Intention Style }\end{array}$} & \multicolumn{4}{c}{ Precision (\%) } & \multicolumn{1}{c}{ Recall (\%) } & \multicolumn{4}{c}{ F1 Score (\%) } \\
\cline { 2 - 6 } & (1) & (2) & (3) & (4) & (5) & (6) & (1) & (2) & (3) & (4) & (5) & (6) & (1) & (2) & (3) & (4) & (5) & (6) \\
\hline Attack & 89.6 & 87.9 & 88.2 & 84.8 & 83.8 & 78.4 & 91.7 & 90.0 & 89.7 & 87.3 & 86.3 & 80.0 & 90.6 & 89.0 & 89.0 & 86.0 & 85.1 & 79.2 \\
\hline Penetration & 97.0 & 95.3 & 93.8 & 92.7 & 92.0 & 84.5 & 95.7 & 94.7 & 95.3 & 92.7 & 91.7 & 87.3 & 96.3 & 95.0 & 94.5 & 92.7 & 91.9 & 85.9 \\
\hline Surveillance & 96.3 & 95.3 & 95.3 & 91.3 & 90.9 & 82.7 & 95.3 & 94.7 & 95.0 & 90.7 & 89.7 & 86.3 & 95.8 & 95.0 & 95.2 & 91.0 & 90.3 & 84.5 \\
\hline Reconnaissance & 93.0 & 91.7 & 93.8 & 88.5 & 88.1 & 83.8 & 97.0 & 95.3 & 96.0 & 92.3 & 91.0 & 84.7 & 95.0 & 93.5 & 94.9 & 90.4 & 89.5 & 84.2 \\
\hline Feint & 90.8 & 88.7 & 88.6 & 87.4 & 86.4 & 80.4 & 88.7 & 86.0 & 85.7 & 83.3 & 84.7 & 76.3 & 89.7 & 87.3 & 87.1 & 85.3 & 85.5 & 78.3 \\
\hline Retreat & 98.3 & 98.0 & 98.0 & 96.7 & 96.3 & 96.0 & 98.7 & 98.7 & 98.7 & 97.0 & 96.7 & 95.0 & 98.5 & 98.3 & 98.3 & 96.8 & 96.5 & 95.5 \\
\hline Electronic interference & 97.3 & 96.2 & 96.6 & 94.5 & 94.2 & 90.2 & 95.0 & 93.7 & 94.0 & 92.3 & 91.3 & 86.0 & 96.1 & 95.0 & 95.3 & 93.4 & 92.7 & 88.1 \\
\hline
\end{tabular}

From Tables 11 and 12 and Figure 15, we can see that Attention-TCN-BiGRU is the most superior, with high accuracy and low loss value low. The accuracy, recall and F1 Score for each type of intention recognition are also the highest, which indicates that combining the Attention, TCN and BiGRU networks can indeed improve the network's ability to recognize intention. TCN is also significantly better than BiGRU, indicating that the recently developed TCN networks are more capable of learning data characteristics. The lowest accuracy rate is for attack intention, which is due to the fact that some of the feint intention samples and surprise intention samples have similar characteristics to attack intention samples, resulting in too many feint and surprise samples being identified as false positives. The lowest recall rate for feint intention is due to the fact that feint intention is too deceptive, and the data characteristics are less easy to learn compared to feint intention. 
The highest accuracy and recall rate was for retreat intentions, which were relatively easy to learn due to the distinctive characteristics of retreat intention.

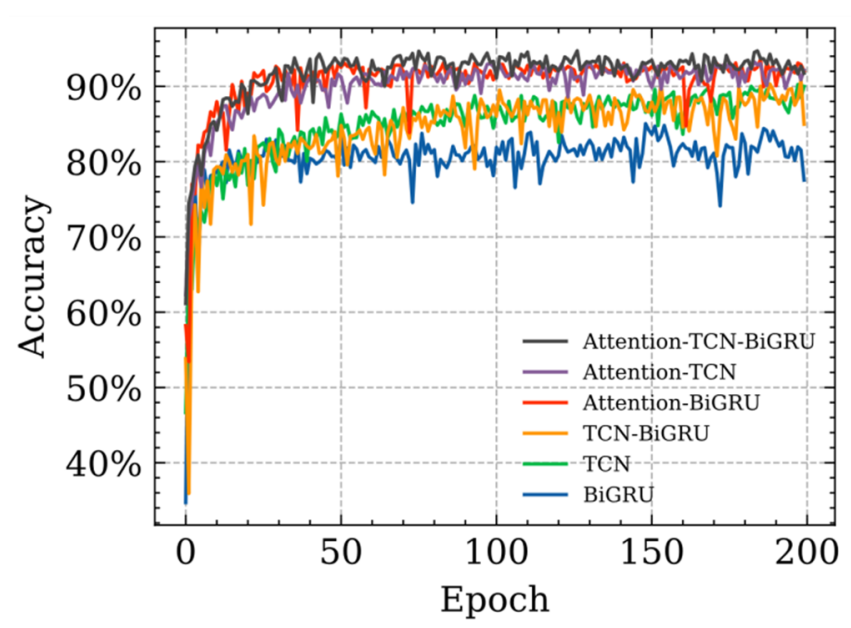

(a)

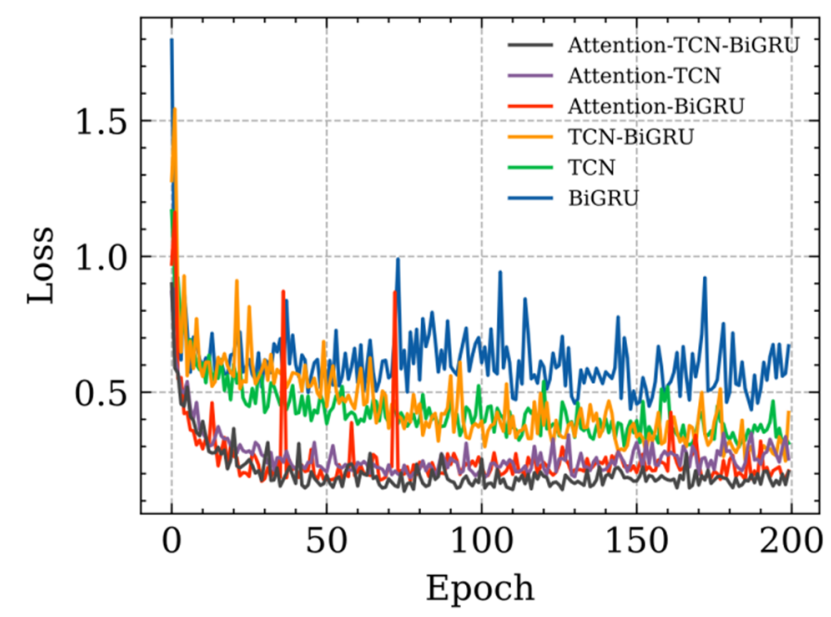

(b)

Figure 15. Changes in accuracy and loss in ablation experiments: (a) Accuracy rate of ablation experiment; (b) loss value of ablation experiment.

\section{Conclusions}

A temporal network for improving intention recognition is designed, which addresses the important role of air target combat intention recognition in modern air warfare. Firstly, by analyzing the combat characteristics of air targets, 12 easily accessible characteristics focusing on both the combat mission and the air posture are extracted. Moreover, the nonnumerical characteristics of the 12 characteristics are numerically processed. Secondly, the Attention mechanism is used to attach attention weights to the various characteristics; the TCN network is used to extract potential characteristics from the data, and the longterm dependencies present in the sequence are captured by the BiGRU network. Finally, generic simulation experiments are conducted to verify the intention recognition capability of our proposed model.

The higher intention recognition accuracy of our proposed Attention-TCN-BiGRU generic is validated in comparison with DBP [11], BN-FC-RES [12], RBF [13], SAE [14], LSTM [16], and PCLSTM [17] methods as well as its ablation experiments. It compares well with other methods in its ability to perform the task of intent recognition of air targets, and it has theoretical implications and reference value for combat systems that aid decision-making.

We will focus on two issues in our future work. The first is the problem of recognizing intentions with high characteristic similarity and high deceptiveness. The second is how to effectively recognize intentions when they change in the detected time series.

Author Contributions: Conceptualization, F.T. and Y.S.; methodology, F.T.; software, F.T.; validation, X.G.; formal analysis, F.T.; investigation, Y.S.; resources, Y.S.; data curation, X.G.; writing—original draft preparation, F.T.; writing-review and editing, F.T.; visualization, F.T.; supervision, Y.S.; funding acquisition, Y.S. All authors have read and agreed to the published version of the manuscript.

Funding: This research was funded by the National Natural Science Foundation of China (under Grants No. 61703426); Young Talent fund of University Association for Science and Technology in Shaanxi, China, under Grant No. 2019038, and the Innovation Capability Support Plan of Shaanxi, China, under Grant No. 2020KJXX-065.

Institutional Review Board Statement: Not applicable.

Informed Consent Statement: Not applicable. 
Data Availability Statement: Not applicable.

Conflicts of Interest: The authors declare no conflict of interest.

\section{Appendix A}

Table A1. Detailed description of seven intention types.

\begin{tabular}{|c|c|}
\hline Intention Type & Description \\
\hline Attack & $\begin{array}{c}\text { Attack ground targets by launching bullets, missiles, and bombs from } \\
\text { aerial targets. }\end{array}$ \\
\hline Penetration & $\begin{array}{l}\text { Use earth curvature and terrain as cover to effectively avoid various } \\
\text { threats and achieve sudden attacks on targets. }\end{array}$ \\
\hline Surveillance & $\begin{array}{l}\text { Passively monitor the situation in a certain space through aerial targets, } \\
\text { usually continuously. }\end{array}$ \\
\hline Reconnaissance & $\begin{array}{c}\text { Actively detect a certain space through air targets, which can be } \\
\text { continuous or intermittent. }\end{array}$ \\
\hline Feint & $\begin{array}{l}\text { A military means to deceive the enemy. Only make a limited attack, } \\
\text { making the enemy's judgment wrong. }\end{array}$ \\
\hline Retreat & Withdraw from the battlefield or conflict. \\
\hline $\begin{array}{l}\text { Electronic } \\
\text { interference }\end{array}$ & $\begin{array}{l}\text { Air targets interfere with radar and communication systems by carrying } \\
\text { electronic jamming equipment. }\end{array}$ \\
\hline
\end{tabular}

Table A2. Symbol description.

\begin{tabular}{cc}
\hline Symbol & Meaning \\
\hline $\boldsymbol{Q}$ & Air target combat intent space set \\
$\boldsymbol{I}^{\left(t_{n}\right)}$ & Real-time feature information of air targets at time $t_{n}$ \\
$\boldsymbol{I}_{T}$ & Real-time characteristic information of air targets at $T$ consecutive times from $t_{1}$ to $t_{T}$ \\
$\boldsymbol{C}$ & Air combat capability factor \\
$x_{i r}^{\prime}$ & The $r$-th data in class $i$ air target characteristics \\
$\boldsymbol{X}_{i}$ & Type $i$ air target characteristics \\
$\boldsymbol{u}_{i}$ & Linear layer output vector in HAN \\
$\delta_{i}$ & Importance weight of the $i$-th word \\
$\boldsymbol{s}$ & Sentence vector obtained after weighted average of hierarchical attention network \\
$\alpha_{i, t}$ & The weight of the i-th type feature at time $t$ \\
$\alpha_{t}$ & Importance weight at time $t$ \\
$\alpha_{i}$ & The average value of the weight of the $i$-th type feature \\
$\boldsymbol{R}_{i}$ & The weighted value of the $i$-th type feature output by the Attention mechanism \\
$R F$ & Receptive field \\
$k$ & Kernel size \\
$d$ & Dilation factor \\
$\boldsymbol{x}_{t}$ & Input sequence value at time $t$ \\
$\boldsymbol{r}_{t}$ & Reset gate at time $t$ \\
$\sigma$ & Sigmoid activation function \\
$\odot$ & Hadamard product of the matrix \\
$\boldsymbol{z}_{t}$ & Update gate at time $t$ \\
$\widetilde{\boldsymbol{h}}_{t}$ & Candidate output state at time $t$ \\
$\boldsymbol{h}_{t}$ & Hidden state at time $t$ \\
$\vec{h}_{t}$ & BiGRU forward hidden layer state at time $t$ \\
$\overleftarrow{\boldsymbol{h}_{t}}$ & Bir target intent type label \\
$y$ &
\end{tabular}

\section{References}

1. Yao, Q.K.; Liu, S.J.; He, X.Y.; Ou, W. Research and Prospect of Battlefield Target Operational Intention Recognition. J. Command. Control 2017, 3, 127-131. [CrossRef] 
2. Xia, P.; Chen, M.; Zou, J.; Feng, X. Prediction of Air Target Intention Utilizing Incomplete Information; Springer: Singapore, 2016. [CrossRef]

3. Sun, Y.L.; Bao, L. Study on Recognition Technique of Targets' Tactical Intentions in Sea Battle Field based on D-S Evidence Theory. Ship Electron. Eng. 2012, 32, 48-51. [CrossRef]

4. Zhao, F.J.; Zhou, Z.J.; Hu, C.; Wang, L.; Liu, T. Aerial Target Intention Recognition Approach based on Belief-Rule-Base and Evidential Reasoning. Electron. Opt. Control 2017, 24, 15-19, 50. [CrossRef]

5. Chen, Y.M.; Li, Y.C. Simulation of Target Tactical Intention Recognition Based on Knowledge Map. Comput. Simul. 2019, 36, 1-4, 19. [CrossRef]

6. Yin, X.; Zhang, M.; Chen, M.Q. Combat Intention Recognition of the Target in the Air Based on Discriminant Analysis. J. Proj. Rocket. Missiles Guid. 2018, 38, 46-50. [CrossRef]

7. Xu, Y.; Cheng, S.; Zhang, H.; Chen, Z. Air Target Combat Intention Identification Based on IE-DSBN. In Proceedings of the 2020 International Workshop on Electronic Communication and Artificial Intelligence (IWECAI), Shanghai, China, $12-14$ June 2020. [CrossRef]

8. Xu, X.M.; Yang, R.; Fu, Y. Situation assessment for air combat based on novel semi-supervised naive Bayes. J. Syst. Eng. Electron. 2018, 29, 768-779. [CrossRef]

9. Qing, J.; Xiantai, G.; Weidong, J.; Nanfang, W. Intention recognition of aerial targets based on Bayesian optimization algorithm. In Proceedings of the 2017 2nd IEEE International Conference on Intelligent Transportation Engineering (ICITE), Singapore, 1-3 September 2017. [CrossRef]

10. Zhou, T.G.; Chen, M.; Wang, Y.H.; He, J.L.; Yang, C.G. Information Entropy Based Intention Prediction of Aerial Targets under Uncertain and Incomplete Information. Entropy 2020, 22, 279. [CrossRef]

11. Zhou, W.W.; Yao, P.Y.; Zhang, J.Y.; Wang, X.; Wei, S. Combat Intention Recognition Foraerial Targets based on Deep Neural Network. Acta Aeronaut. Astronaut. Sinca 2018, 39, 322468. [CrossRef]

12. Zhai, X.Y.; Yang, F.B.; Ji, L.N.; Li, S.Q.; Lv, H.L. Air Combat Targets Threat Assessment based on Standardized Fully Connected Network and Residual Network. Fire Control Command Control 2020, 45, 39-44. [CrossRef]

13. Liu, Y.J.; Kou, G.H.; Song, J.H. Target Recognition based on RBF Neural Network. Fire Control Command Control 2015, 40, 9-13. [CrossRef]

14. Ou, W.; Liu, S.J.; He, X.Y.; Guo, S.M. Tactical Intention Recognition Algorithm based on Encoded Temporal Features. Command Control Simul. 2016, 38, 36-41. [CrossRef]

15. Ou, W.; Liu, S.J.; He, X.Y.; Cao, Z.G. Study on Intelligent Recognition Model of Enemy Target's Tactical Intention on Battlefield. Comput. Simul. 2017, 34, 10-14, 19. [CrossRef]

16. Liu, Z.D.; Chen, M.; Wu, Q.X.; Chen, S.D. Prediction of Unmanned Aerial Vehicle Target Intention Under Incomplete Information. Sci. Sin. Inf. 2020, 50, 704-717. [CrossRef]

17. Xue, J.; Zhu, J.; Xiao, J.; Tong, S.; Huang, L. Panoramic Convolutional Long Short-Term Memory Networks for Combat Intention Recognition of Aerial Targets. IEEE Access 2020, 8, 183312-183323. [CrossRef]

18. Bai, S.; Kolter, J.Z.; Koltun, V. An Empirical Evaluation of Generic Convolutional and Recurrent Networks for Sequence Modeling. arXiv 2018, arXiv:1803.01271.

19. Bi, J.; Zhang, X.; Yuan, H.; Zhang, J.; Zhou, M. A Hybrid Prediction Method for Realistic Network Traffic With Temporal Convolutional Network and LSTM. IEEE Trans. Autom. Sci. Eng. 2021. [CrossRef]

20. Yan, J.; Liu, J.; Yu, Y.; Xu, H. Water Quality Prediction in the Luan River Based on 1-DRCNN and BiGRU Hybrid Neural Network Model. Water 2021, 13, 1273. [CrossRef]

21. Lorenc, A.; Burinskiene, A. Improve the orders picking in e-commerce by using WMS data and BigData analysis. FME Trans. 2021, 49, 233-243. [CrossRef]

22. Lorenc, A.; Kunar, M. The most common type of disruption in the supply chain-Evaluation based on the method using artificial neural networks. Int. J. Shipp. Transp. Logist. 2021, 13, 1-24. [CrossRef]

23. Lu, G.Y.; Ding, Y.Y. Study on Intention Recognition to Foe of Underwater Platform. Command Control Simul. 2012, 34, 100-102.

24. Chen, H.; Ren, Q.L.; Hua, Y.; Qiu, Y.N. Fuzzy Neural Network based Tactical Intention Recognition for Sea Targets. Syst. Eng. Electron. 2016; 38, 1847-1853. [CrossRef]

25. Kojadinovic, I.; Marichal, J.L. Entropy of bi-capacities. Eur. J. Oper. Res. 2007, 178, 168-184. [CrossRef]

26. Xi, Z.F.; Xu, A.; Kou, Y.X.; Li, Z.W.; Yang, A.W. Target threat assessment in air combat based on PCA-MPSO-ELM algorithm. Acta Aeronaut. AstroNautica Sin. 2020, 41, 323895. [CrossRef]

27. Zhai, X.Y.; Yang, F.B.; Ji, L.N.; Lv, H.L.; Bai, Y.Q. Standardized Fully Connected Network and Residual Network Model for Combat Intention Analysis of Air Targets. Foreign Electron. Meas. Technol. 2019, 38, 1-6. [CrossRef]

28. Kulak, O. A decision support system for fuzzy multi-attribute selection of material handling equipments-ScienceDirect. Expert Syst. Appl. 2005, 29, 310-319. [CrossRef]

29. Anderson, P.; He, X.; Buehler, C.; Teney, D.; Johnson, M.; Gould, S.; Zhang, L. Bottom-Up and Top-Down Attention for Image Captioning and Visual Question Answering. arXiv 2017, arXiv:1707.07998.

30. Luong, M.T.; Pham, H.; Manning, C.D. Effective Approaches to Attention-based Neural Machine Translation. Comput. Sci. 2015. [CrossRef] 
31. Vaswani, A.; Shazeer, N.; Parmar, N.; Uszkoreit, J.; Jones, L.; Gomez, A.N.; Kaiser, Ł.; Polosukhin, I. Attention is All You Need. arXiv 2017, arXiv:1706.03762.

32. Wang, W.; Sun, Y.X.; Qi, Q.J.; Meng, X.F. Text Sentiment Classification Model based on BiGRU-attention Neural Network. Appl. Res. Comput. 2019, 36, 3558-3564. [CrossRef]

33. Yang, Z.; Yang, D.; Dyer, C.; He, X.; Smola, A.; Hovy, E. Hierarchical Attention Networks for Document Classification. In Proceedings of the 2016 Conference of the North American Chapter of the Association for Computational Linguistics: Human Language Technologies, San Diego, CA, USA, 12-17 June 2016. [CrossRef]

34. Song, Y.; Gao, S.; Li, Y.; Jia, L.; Li, Q.; Pang, F. Distributed Attention-Based Temporal Convolutional Network for Remaining Useful Life Prediction. IEEE Internet Things J. 2020, 8, 9594-9602. [CrossRef]

35. Fan, Y.Y.; Li, C.J.; Yi, Q.; Li, B.Q. Classification of Field Moving Targets Based on Improved TCN Network. Comput. Eng. 2021, 47, 106-112. [CrossRef]

36. Gao, Y.; Wang, W.; Lin, Q.; Cai, F.; Chai, Q. Fault Diagnosis for Power Converters Based on Optimized Temporal Convolutional Network. IEEE Trans. Instrum. Meas. 2020, 70. [CrossRef]

37. Huang, Q.; Hain, T. Improving Audio Anomalies Recognition Using Temporal Convolutional Attention Network. In Proceedings of the ICASSP 2021-2021 IEEE International Conference on Acoustics, Speech and Signal Processing (ICASSP), Toronto, ON, Canada, 6-11 June 2021. [CrossRef]

38. Shi, H.; Wang, L.; Scherer, R.; Woźniak, M.; Zhang, P.; Wei, W. Short-term Load Forecasting Based on AdaBelief Optimized Temporal Convolutional Network and Gated Recurrent Unit Hybrid Neural Network. IEEE Access 2021, 9, 66965-66981. [CrossRef]

39. Zhu, R.; Liao, W.; Wang, Y. Short-term prediction for wind power based on temporal convolutional network. Energy Rep. 2020, 6, 424-429. [CrossRef]

40. Xu, Z.; Zeng, W.; Chu, X.; Cao, P. Multi-Aircraft Trajectory Collaborative Prediction Based on Social Long Short-Term Memory Network. Aerospace 2021, 8, 115. [CrossRef]

41. Liu, Y.; Ma, J.; Tao, Y.; Shi, L.; Wei, L.; Li, L. Hybrid Neural Network Text Classification Combining TCN and GRU. In Proceedings of the 2020 IEEE 23rd International Conference on Computational Science and Engineering (CSE), Guangzhou, China, 29 December-1 January 2020. [CrossRef]

42. Sun, Y.C.; Tian, R.L.; Wang, X.F. Emitter signal recognition based on improved CLDNN. Syst. Eng. Electron. 2021, 43, 42-47. [CrossRef]

43. Chen, J.X.; Jiang, D.M.; Zhang, Y.N. A Hierarchical Bidirectional GRU Model With Attention for EEG-Based Emotion Classification. IEEE Access 2019, 7, 118530-118540. [CrossRef]

44. Kiefer, J.; Wolfowitz, J. Stochastic Estimation of the Maximum of a Regression Function. Ann. Math. Stat. 1952, 23, 462-466. [CrossRef]

45. Tieleman, T.; Hinton, G. Lecture 6.5-rmsprop: Divide the Gradient by a Running Average of its Recent Magnitude. COURSERA Neural Netw. Mach. Learn. 2012, 4, 26-30.

46. Kingma, D.; Ba, J. Adam: A Method for Stochastic Optimization. arXiv 2014, arXiv:1412.6980.

47. Dozat, T. Incorporating Nesterov Momentum into Adam. 2016. Available online: https://openreview.net/forum?id=OM0jvwB8 jIp57ZJjtNEZ (accessed on 28 September 2021).

48. Xi, Z.F.; Xu, A.; Kou, Y.X.; Li, Z.W.; Yang, A.W. Air Combat Threat Assessment Based on Improved GRA-TOPSIS. J. Beijing Univ. Aeronaut. Astronaut. 2020, 46, 388-397. [CrossRef]

49. Yang, A.W.; Li, Z.W.; Xu, A.; Xi, Z.F.; Chang, Y.Z. Threat Level Assessment of the air Combat target Based on Weighted Cloudy Dynamic Bayesian Networks. Flight Dyn. 2020, 38, 87-94. [CrossRef] 